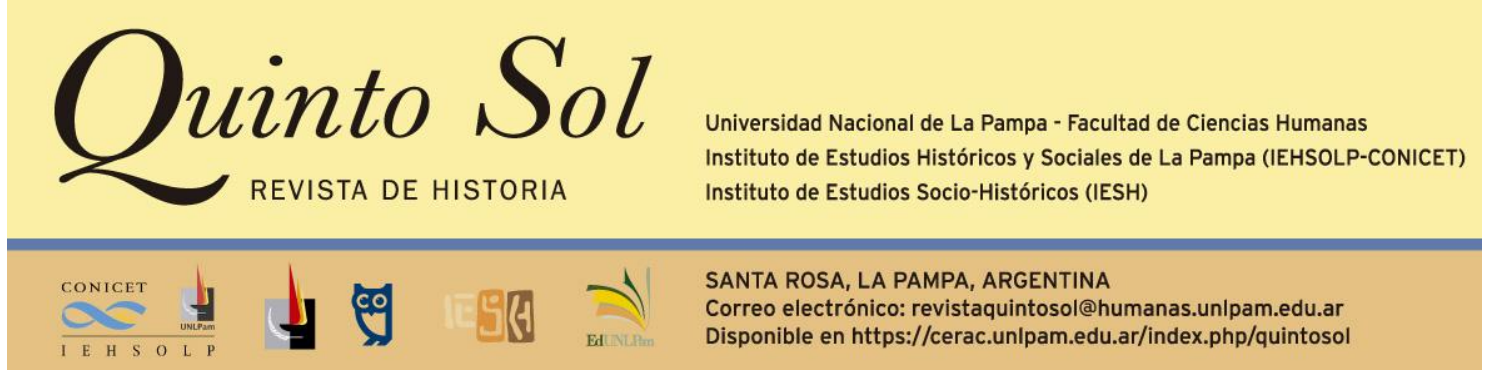

Quinto Sol, vol. 26, no 1, enero-abril 2022, ISSN 1851-2879, pp. 1-23

http://dx.doi.org/10.19137/qs.v26i1.5834

Esta obra se publica bajo licencia Creative Commons 4.0 Internacional. (Atribución-No ComercialCompartir Igual)

\title{
Negocios catalanes, capitales argentinos: una red transnacional al fondo del primer peronismo
}

\author{
Catalan businesses and Argentinian capitals. A transnational \\ network behind first Peronism
}

\section{Negócios catalães, capitais argentinos: uma rede transnacional no fundo do primeiro peronismo}

\section{Enrique Faes Díaz}

Universidad Complutense de Madrid

España

Correo electrónico: efaes@ucm.es

\section{Resumen}

Este trabajo identifica y caracteriza una red corporativa de intereses argentinos y españoles (catalanes en su mayoría) tejida en torno a una docena de empresas radicadas en Buenos Aires en la primera mitad del siglo XX. A partir del núcleo directivo de la sociedad Providencia Compañía Argentina de Seguros, se localizan nodos de confluencia de distintos grupos empresariales, se analizan estrategias corporativas y se calibra el grado de compromiso de sus integrantes con opciones políticas disponibles en el momento, en España y en Argentina.

Palabras clave empresarios red corporativa poder político peronismo 


\section{Abstract}

This paper identifies and characterizes a corporate network based on Argentinian-Spanish business (Catalan mainly) that was built around a dozen companies all located in Buenos Aires, in the first half of 20th century. Studying the board of directors of the company Providencia Compañía Argentina de Seguros, it finds meeting points in which different groups are involved and it analyzes what corporate strategies they applied. It also determines whether any political compromise was acquired by the members of the network, both in Spain and Argentina.

\section{Resumo}

Este resumo identifica e caracteriza uma rede corporativa de interesses argentinos e espanhóis (na sua maioria catalães) em torno a uma dúzia de empresas radicadas em Buenos Aires na primeira metade do século XX. A partir do núcleo diretivo da sociedade Providencia Companhia Argentina de Seguros, localizam-se nodos de confluência de diferentes grupos empresariais, analisam-se estratégias corporativas e calibra-se o grau de compromisso de seus integrantes com opções políticas disponíveis nesse momento, na Espanha e na Argentina.

\section{Keywords}

businessmen

corporate network political power

Peronism 


\section{Negocios catalanes, capitales argentinos: una red transnacional al fondo del primer peronismo}

\section{Introducción ${ }^{1}$}

En vísperas de la Segunda Guerra Mundial, se calcula que entre 20.000 y 30.000 emigrantes españoles procedentes de Cataluña se habían asentado en la ciudad de Buenos Aires (Campins y Pfeiffer, 2011; Fernández, 2019). No fueron tantos como los naturales de Galicia ni lograron equilibrar el predominio de migrantes de la mitad norte de España. Su peso demográfico se ha estimado en poco más de un $10 \%$ del total de españoles radicados en la ciudad. Pero un par de singularidades contribuyeron a hacerlos más visibles sobre otras colectividades: la respuesta fragmentada a la formulación de una identidad nacional específicamente catalana a finales del siglo XIX (agudizada por una recepción dispar de la Segunda República española en 1931 y el inicio de una guerra civil en 1936$)^{2}$ y una presencia activa en el ámbito de los negocios.

Calibrar el alcance de esto último es algo que ha preocupado a la historiografía económica, puesto que Cataluña lideró el despliegue de la industria en España hasta 1930, y mantuvo más allá su empuje como gran vivero de empresas del país (Puig, 2006). Fue, por tanto, la "primera región industrial española", por cronología y por impulso (Carreras, 1990), y de ella emanó una cultura corporativa de la que se ha llegado a inferir que "la historia contemporánea de Cataluña es, por encima de todo, la historia de sus empresas y de sus empresarios" (Maluquer, 2006, p. 9). En clave transnacional, esta singularidad permitió que los migrantes catalanes arribaran a Buenos Aires con una cualificación profesional ventajosa. Pudo estimular la idea de crear nuevas sociedades en destino a partir de modelos previos, y fraguó la imagen del catalán radicado en Argentina como alguien, en general, dotado de una "cierta capacidad para la industria y los negocios" (Campins y Pfeiffer, 2011, p. 19). Una constelación de pequeños empresarios, comerciantes, profesionales y empleados sustentó las dos grandes asociaciones de catalanes en Buenos Aires, el Centre y el Casal Catalá, fundadas respectivamente en 1886 y 1908. Y no escaseó presencia catalana en

\footnotetext{
${ }^{1}$ Este trabajo es fruto de una estancia de investigación adscrita al Instituto de Historia Argentina y Americana "Dr. Emilio Ravignani" (UBA-Conicet), ubicado en la ciudad de Buenos Aires. Quiero agradecer en particular a Andrea Lluch y Claudio Belini las sugerencias y orientaciones recibidas en el transcurso de la estancia, y a Sergio Riesco por sus observaciones posteriores sobre la estructura del texto. El presente artículo se inserta, además, en el proyecto de investigación "Territorios de la memoria: otras culturas, otros espacios en Iberoamérica. Siglos XX y XXI", con sede de trabajo en la Facultad de Ciencias Políticas y Sociología de la Universidad Nacional de Educación a Distancia (Madrid, España), denominado PID2020$113492 R B-100$.

2 La mayoría de los trabajos al respecto adoptan perspectivas políticas y/o culturales para indagar mecanismos de sociabilidad: Jensen (2010); Yrurzun (2017); Fernández (2019); Ferreyra (2020); Lucci (2020). Hay también obras indiciarias o autobiográficas, y aproximaciones biográficas: Monner (1927); Rocamora (1992, 1995); Manent (1992); Sarramone (2004). Entre los estudios migratorios más generales citaremos: Moya (2004); García Sebastiani (2010); Núñez Seixas (2014).
} 
la dirección de la Cámara Española de Comercio en la República Argentina, creada en 1888 y reactivada con el cambio de siglo, cuando España fortaleció su comercio hacia el Río de la Plata tras perder el dominio sobre Cuba (Fernández, 2004, 2019; Vidal, 2011).

En el período de entreguerras se consolidó el vínculo. El caso de la Compañía Hispano Americana de Electricidad (CHADE, cuarta empresa española por volumen de activos y principal inversión de ese país en América del Sur) prueba que equipos de businessmen catalanes asentados en Buenos Aires fueron mucho más allá de un rango de negocios medianos, en conexión además con intereses multinacionales amplios. ${ }^{3}$ Para ello se valieron del anclaje proporcionado por la propia corriente migratoria, primero, y tejieron luego una relación más elaborada desde la Casa de América de Barcelona, una asociación fundada en 1911 que funcionó como think tank del empresariado catalán con intereses en Sudamérica. Bajo inspiración del abogado, financiero y político Francesc Cambó i Batlle, esa entidad potenció el flujo de información, personas, productos y capitales entre las redes corporativas catalana y argentina, en un momento propicio para la inversión extranjera en este último país (Dalla Corte, 2005; Lanciotti y Lluch, 2018).

La Segunda Guerra Mundial alteró el escenario, lo que, como es sabido, enrareció el comercio y las relaciones internacionales. La dictadura de Francisco Franco en España, aislada hasta los inicios de la Guerra Fría, halló su principal socio comercial en la Argentina del presidente Juan Domingo Perón (1946-1955). Pero el vínculo fue asimétrico y, además, breve: hacia 1950, el enfriamiento de la economía argentina y la aproximación de Estados Unidos a Franco clausuraron apenas un lustro de expectativas mutuas (Figallo, 1992; Rein, 2003). Explorar qué ocurrió a la sombra de los acuerdos Franco-Perón en el ámbito de la iniciativa privada es el propósito central de este trabajo, en plena transición argentina de un ciclo de inversión y endeudamiento basado en el comercio de exportación a otro centrado en la industria. Lejos del dinamismo acreditado, por ejemplo, por la comunidad italiana, la segunda posguerra pareció prolongar un período de cierta hibernación de los intereses empresariales españoles en Argentina, a la luz de las cifras de radicación de empresas para el período 1944-1959 (Lanciotti y Lluch, 2018). Tampoco habrían emergido nuevos grupos empresariales ${ }^{4}$ en ese intervalo, ni argentinos ni hispano-argentinos, con ambos países centrados en culminar la construcción de sendas redes corporativas cada vez más estables y centralizadas (Barbero, 2011; Rubio y Garrués, 2016, 2018; Lluch y Salvaj, 2018). Todo ello acompañado, para los actores del universo corporativo local, por la acentuación de la diversificación, un crecimiento económico notable (aunque no lineal), una mejor distribución del ingreso y, muy en especial, la expansión del mercado interno (Belini y Korol, 2020; Lanciotti, 2021).

La escasez de trabajos específicos para este momento de aparente degradación del tejido empresarial hispano-argentino permitirá indagar si esa colectividad catalana, señalada por su especialización en los negocios, mantuvo o no su empuje transnacional

\footnotetext{
3 Sobre la CHADE pueden consultarse Cámara de Diputados de la Nación (1941); Alcalde (2006); Dalla Corte (2006); Lanciotti (2011); Cabrera (2011); De Riquer (2016).

${ }^{4}$ Asumimos la definición de "grupo empresarial" proporcionada por Tarun Khanna y Yishay Yafeh (citado en Barbero, 2011, p. 3): "conjuntos de empresas legalmente independientes, que operan en diversos mercados (a menudo no relacionados entre sí), ligadas entre sí por vínculos persistentes, formales e informales".
} 
en la segunda posguerra, y en qué medida fortaleció conexiones ya existentes o estableció otras nuevas. Para ello tomamos como eje central el año 1947, momento de la desaparición de Cambó como referencia crucial de los negocios catalanes conectados a Argentina y fecha iniciática de la planificación económica peronista. El trabajo se inicia con la exploración de un viaje de negocios que empresarios y técnicos catalanes realizaron a Buenos Aires entre febrero y mayo de 1947. El artículo conecta, hacia atrás, con el golpe militar de junio de 1943, que prefiguró el peronismo al calor de un ciclo económico favorable, y se solapa hacia delante con el primer gobierno de Perón (1946-1951), señalado por su implicación industrializadora (Belini, 2009, 2014). En un segundo apartado se estudia el caso de Providencia Compañía Argentina de Seguros, un negocio financiero a tres países. A continuación, caracterizamos a los miembros más importantes de una red flotante entre los principales conglomerados argentinos y el grupo catalán articulado por Cambó, con el fin de obtener indicadores de su dinamismo emprendedor, discriminar grados de centralidad individual mediante el cruce de directorios y explorar si entre ellos existió algún grado de coherencia ideológica. Cierra el artículo un breve apartado de conclusiones. En cuanto a las fuentes empleadas, el trabajo se basa en el análisis de publicaciones económicas del período y se completa con documentación de archivos familiares e institucionales de España y de Argentina.

\section{Un viaje de negocios (Buenos Aires, 1947)}

En febrero de 1947, un grupo de técnicos de la industria textil catalana desembarcó en Buenos Aires liderado por Demetrio Carceller Segura, un empresario que, incluso, había sido ministro de Industria y Comercio de España entre 1940 y 1945. También era ingeniero textil titulado, de origen catalán. Tras su salida del gobierno, el exministro se replegó a su actividad privada y emprendió un proceso de diversificación mediante participaciones directas en una docena de sectores, con base en el petróleo e inclinación por la industria. Lo que Carceller se proponía al partir hacia Argentina era, de hecho, "ver, conocer y estudiar la potencialidad industrial" de aquel país y, llegado el caso, "fundar grandes empresas." Consciente como empresario del atractivo de derivar inversiones hacia aquel destino (considerado más seguro y estable en relación con la España de la Guerra Civil y con la Europa de la Segunda Guerra Mundial), Carceller además pretendía, según dijo, captar capitales argentinos con los que modernizar la industria española. ${ }^{5}$

Ambos objetivos resultaban compatibles. Por el lado español, la llegada al Ministerio de Industria de Juan Antonio Suanzes, partidario de profundizar la estatalización, prolongó entre el empresariado la incertidumbre sobre la pervivencia de límites a la actividad privada. ${ }^{6}$ Aunque la dictadura templó su retórica nacionalista para incorporar capitales extranjeros, la economía española presentó un panorama más que mediocre, al menos hasta la década de 1950 (Catalan, 1995; Cabrera y Del Rey, 2002; Comín y Martorell, 2013). La situación era más halagüeña en Argentina. El buen

\footnotetext{
${ }^{5}$ De Santiago, Í. (13 de marzo de 1947). Desde Buenos Aires. La visita de don Demetrio Carceller. La Nueva España, p. 3. Hemeroteca de La Nueva España, Oviedo, España; El Adelanto, 2 de febrero de 1947, p. 1. Hemeroteca Digital de la Biblioteca Nacional de España (HDBNE), Madrid, España; Faes (2020).

${ }^{6}$ Para Suanzes, véase Ballestero (1993). Para las tensiones entre Suanzes y Carceller (este último más aperturista), véase San Román (1999) y Faes (2020).
} 
desempeño de su comercio exterior venía impulsando el crecimiento de la renta nacional, la capacidad de inversión, los salarios reales y la idea -en boga ya desde el impacto regional de la crisis de 1929- de fortalecer la industria doméstica mediante una arquitectura general de sustitución de importaciones (Gerchunoff y Llach, 1998; Cortés, 2006; Belini y Korol, 2020).

En su primer ciclo de gobierno, el peronismo apenas reguló el marco en que operaba la iniciativa privada extranjera. Sobre una tradición jurídica laxa respecto de la nacionalidad de empresas y empresarios, no se aumentó el control a la inversión externa ni se legisló en sentido perjudicial para las sociedades no argentinas. Estas pudieron actuar en equidad respecto de las que sí lo eran, incluso después de que en 1948 se sancionara una regulación para orientar la radicación de industrias extranjeras (Lanciotti y Lluch, 2018). Pese a que Carceller declarase a la prensa porteña que no era el momento de aprovechar el capital y la experiencia española para industrializar Argentina, sino de recabar cooperación argentina para emprender el proceso de industrialización de España, la presencia de técnicos textiles en su comitiva sugería lo contrario. Durante su visita se escrituró, de hecho, una empresa textil que pronto lideraría: Sociedad Anónima de Manufacturas Textiles (SADEMA), con participación fundacional, entre otros socios catalanes, de José Bau Nolla, Evaristo Arnús Moraleda, Jesús Raventós Fatjó, Crisanto Ollé Pérez o Andrés Bausili Sanromá. ${ }^{7}$ Este último, hombre de confianza de Cambó en Buenos Aires y director delegado de la CHADE, presidía además en ese momento la Cámara Española de Comercio, entidad que obsequió a Carceller con un almuerzo en el Jockey Club de la capital porteña durante su visita. Dos años después, el empresario español figuraba como presidente de SADEMA, inscrita para fabricar, comercializar y vender "hilados y tejidos de lana", sin descartar el aprovechamiento de "otras fibras." ${ }^{8}$

La especialización catalana en el sector venía de lejos, puesto que la industrialización pionera en Cataluña fue la historia de una revolución textil al estilo de la británica, aunque con menor disponibilidad de tecnología (Martínez-Galarraga y Prat, 2016). En las primeras décadas del siglo XX, Argentina se convirtió en el principal cliente exterior de esas manufacturas, al tiempo que el puerto de Barcelona alcanzó un papel sustancial en el conjunto de exportaciones españolas hacia Buenos Aires (Fernández, 2004). El patrón general de embarques no parecía haber variado excesivamente tras la Segunda Guerra Mundial. La entrada de tejidos españoles representaba, con diferencia, la principal partida de importaciones argentinas en 1947, y la prevalencia del textil en el flujo comercial desde Barcelona era indiscutible. ${ }^{9}$ Un sondeo en los datos consulares del período muestra que los tejidos de algodón para Buenos Aires concentraron el $80 \%$ del valor total estimado de las operaciones de uno a

\footnotetext{
${ }^{7}$ A ellos nos referiremos en el apartado 4.

${ }^{8}$ Información obtenida de la Base de Datos de Empresas Extranjeras en Argentina/Foreign Companies in Argentina Database BDEEA/FCAD-PICT 2010/0501. Agencia Nacional de Promoción Científica y Tecnológica, Argentina (http://agencia.mincyt.gob.ar/); La Información, octubre de 1947, p. 24. Banco Central de la República Argentina y Biblioteca Tornquist (BCRA-BT), Buenos Aires, Argentina.

${ }^{9}$ Por convenio del 30 de octubre de 1946, España obtuvo un crédito de 350 millones de pesos para adquirir mercancías argentinas, ampliable a otros 400 millones. Un protocolo adicional elevaría el 9 de abril de 1948 el crédito hasta 1.750 millones de pesos, disponibles hasta 1951. Memorias del Banco Central de la República Argentina (1948, pp. 38-39; 1949, pp. 33-54). Banco Central de la República Argentina, Biblioteca Prebisch. La información sobre las importaciones se ha extraído de tablas con datos oficiales reproducidas en Cámara Española de Comercio en la República Argentina (1948, pp. 11-16). BCRA-BT.
} 
otro puerto en marzo de 1947, cuando la misión comandada por Carceller inició sus prospecciones. $Y$ ese porcentaje se eleva al $89 \%$ si incluimos el resto de materias textiles (lana, seda o rayón) y algún suministro menor de maquinaria. Las cifras son similares para los meses anteriores. No fue infrecuente encontrar vapores que partieran de la capital catalana con tejidos de algodón que sumaban hasta el 95\% del valor de todo lo que llevaban en sus bodegas, para regresar en cambio a España repletos de alimentos. $^{10}$

Cuadro 1. Diez mercancías principales exportadas a Argentina desde el puerto de Barcelona en marzo de 1947 (vapores María O., Cabo de Hornos y Río Neuquén), según valor estimado

\begin{tabular}{|l|c|c|}
\hline Producto & Cantidad (en $\mathrm{kg}$ ) & Valor estimado (en pesetas) \\
\hline 1. Tejidos de algodón & 268.240 & 12.979 .052 \\
\hline 2. Tejidos de rayón mezcla algodón & 7.855 & 490.977 \\
\hline 3. Pelo de conejo & 4.820 & 395.500 \\
\hline 4. Ropa y efectos personales usados & 18.544 & 335.500 \\
\hline 5. Papel para cigarrillos & 14.406 & 275.627 \\
\hline 6. Tejidos de rayón & 3.441 & 273.634 \\
\hline 7. Libros científicos & 10.405 & 272.145 \\
\hline 8. Ropa y efectos usados & 11.794 & 260.000 \\
\hline 9. Almendra en pepita & 9.000 & 168.500 \\
\hline 10. Maquinaria textil & 1.650 & 81.021 \\
\hline TOTAL DEL MES & $\mathbf{3 9 9 . 2 3 1}$ & $\mathbf{1 6 . 1 0 4 . 5 1 4}$ \\
\hline
\end{tabular}

Fuente: Elaboración propia a partir de planillas del Consulado General de la República Argentina en Barcelona. SRDC, Subsección 62, Embajada en Madrid II, Caja AH/0001.

El propio Cambó participaba en una potente empresa textil de capital parcialmente catalán, Formio Argentino, fundada en 1931 bajo los auspicios de la familia Güell, una de las más emblemáticas en el sector desde finales del XIX. A esta firma nos referiremos más adelante. En la década de 1940, el margen de expansión para la industria textil argentina era amplio, y su desarrollo, prometedor. La rama lanera acumulaba ventaja, ya que abastecía la casi totalidad de la demanda interna en vísperas de la guerra. La manufactura del algodón ocupaba un segundo plano, pero se expandía notablemente. Esa diferencia se redujo en los primeros años de posguerra, con resultados distintos a los planteados por el Estado para ambas ramas, y gracias, al menos en parte, a que el sector textil concentró la mayor proporción del crédito público a la industria hasta la caída de Perón en 1955 (Rougier, 2007; Belini, 2010, 2014). Por su lado, empresarios e inversores hallaron oportuno colocar capitales en actividades industriales (la Bolsa de Comercio de Buenos Aires registró en 1947 un máximo de transacciones de títulos ligados a la industria, con casi 1.000 millones de pesos movilizados) ${ }_{1}^{11}$ y debieron definirse ante la apertura de oportunidades.

¿Quiénes fueron los interlocutores locales de Carceller y su comitiva? Con las fuentes disponibles, apenas podemos consignar que el exministro español admitió

\footnotetext{
${ }^{10}$ Las cifras son producto de elaboración propia a partir de Planillas de exportación de mercaderías con destino a la Argentina por el puerto de Barcelona, diciembre 1946-marzo 1947. Sección Representaciones Diplomáticas y Consulares (SRDC), Subsección 62, Embajada en Madrid II, Caja AH/0001. Archivo Histórico de Cancillería, Ciudad Autónoma de Buenos Aires, Argentina.

${ }^{11}$ Cámara Española de Comercio en la República Argentina (1948, p. 18).
} 
haberse entrevistado con "las personalidades más eficientes y decisivas de las esferas económicas argentinas." ${ }^{12}$ Decir esto en 1947 equivalía a señalar a dos funcionarios de alto rango: Miguel Miranda Forcada y José Figuerola Tressols. El primero era el presidente del Banco Central -a cuyo alrededor orbitaba un sistema financiero nacionalizado- y responsable clave de las negociaciones económicas internacionales hasta el debilitamiento de su posición política a mediados de año. Empresario selfmade, considerado por Perón por su competencia técnica y su concisión en asuntos económicos, Miranda no perteneció "ni por su origen ni por su comportamiento" a la cúpula empresarial más ortodoxa de su país (Belini, 2013, p. 246). Su perfil fue similar al que Carceller había mantenido en España junto a Franco. En cuanto a Figuerola, secretario técnico con rango de ministro, fue el hombre de confianza de Perón para diseñar el primer Plan Quinquenal, en un marco de progresiva reglamentación sobre la actividad económica. Figuerola se había forjado profesionalmente en la administración del general Miguel Primo de Rivera en España (1923-1930). Era catalán, y antes de establecerse en Buenos Aires había sido profesor de Derecho en la Escuela Social de Barcelona, una entidad concebida por el régimen de Primo, dictatorial y corporativo, para formar cuadros administrativos en la gestión pública (Berrotarán, 2013).

En el imaginario de emprendedores catalanes que decidían cruzar el océano, acceder a estos dos funcionarios podía resultar determinante. Viajaban movidos por las "facilidades concedidas por el Plan quinquenal. Por ser el país de mayores posibilidades de exportación. Facilidad comercial entre España y Argentina. Afinidad de raza y deseos de técnicos y obreros especializados de venir a radicar al país." ${ }^{13}$ Pero se exponían, como sucedió en algún caso, a vagar varios meses sin poder recuperar de la aduana el muestrario que llevaban consigo, y a la confusión de "no encontrar el Organismo competente" al que dirigirse. La movilidad fue alentada en todo caso por la delegación diplomática argentina en España. Y en el caso catalán hubo una apuesta decidida por imbricar el desarrollo de la industria textil de ambos países. El Gobierno argentino instruyó a sus funcionarios para que adquirieran maquinaria catalana, y el propio embajador se desplazó a Sabadell, "centro de extraordinaria importancia textil," en cuyas fábricas -dijo- "pude apreciar el grado de adelanto de las mismas y la labor social obrera que se cumple en ellas.. ${ }^{14}$

En este contexto empático, es posible rastrear quiénes fueron los partners locales de los empresarios catalanes en ese momento. Para ello nos detendremos en la única empresa porteña respecto de la que consta una vinculación anterior (aunque indirecta) del líder de la comitiva de 1947, Providencia Compañía Argentina de Seguros, filial desde 1943 de la española Compañía Hispano Americana de Seguros y Reaseguros (CHASYR). En la medida en que el negocio asegurador está conectado con intereses financieros, y habilita altos niveles de internacionalización de capitales, el ejercicio permitirá desplegar una red empresarial tendida por confluencia entre ambos países.

\footnotetext{
12 De Santiago, Í. (13 de marzo de 1947). Desde Buenos Aires. La visita de don Demetrio Carceller. La Nueva España, p. 3.

${ }^{13}$ Carta de Fermín Segarra Canalda, apoderado de la empresa catalana Mobba, a Luis [sic] Figuerola. Buenos Aires, 3 de marzo de 1947. Secretaría de Asuntos Técnicos, Correspondencia, Caja 696/47. Archivo Intermedio del Archivo General de la Nación (AI-AGN), Buenos Aires, Argentina.

14 Cartas del embajador Pedro Radío al ministro de Asuntos Exteriores. Madrid, 11 y 22 de julio de 1947. SRDC, Subsección 62, Caja AH/0002.
} 


\section{Providencia, CHASYR y Generali: un vínculo triangular}

Con la misión catalana comenzando su viaje de negocios, una publicación porteña dedicó un par de páginas a elogiar la buena marcha de la aseguradora Providencia. ${ }^{15}$ Esta había duplicado la captación de primas en los últimos ejercicios, hasta rozar los 2,8 millones de pesos. No dejaba de añadir nuevos ramos de actividad. Declaraba un beneficio neto en el último ejercicio de 71.940 pesos y esgrimía una situación solvente, con el $37 \%$ de sus inversiones libres de cargas por reservas y garantías. Todo ello gracias a un vuelco operado en los últimos tres años. Aunque la sociedad funcionaba desde 1904, había sido refundada en la práctica con un desembarco de capitales españoles (catalanes, mayormente) a mediados de 1943, en plena Guerra Mundial. ${ }^{16}$ En ese momento cambiaron sus estatutos, se realizó un canje de acciones y se modificó por completo el organigrama de propietarios y directivos de la empresa, que se convirtió en una filial regional de la firma española CHASYR. Hasta entonces, Providencia operaba solo con seguros de vida. Comenzaba a mostrar débiles indicios de recuperación, pero acumulaba pérdidas de casi 400.000 pesos. Para sanear esa situación, los nuevos propietarios orientaron la compañía hacia el transporte marítimo y dieron entrada a grandes operaciones de reaseguro con compañías de mayor tamaño. Las cifras que Providencia exhibió en su primer ejercicio, tras el cambio de propiedad (que abarcó solo un semestre, el segundo de 1943), sugieren que ese era el plan desde el principio. El 88,5\% de las primas brutas que la compañía ingresó en lo que quedaba del año procedieron de contratos de seguros marítimos. Y casi la totalidad (un 94,27\%) fueron, a su vez, objeto de reaseguro, una práctica extendida en la primera globalización con la que varias compañías acuerdan compartir grandes riesgos para evitar situaciones delicadas en caso de siniestro. ${ }^{17}$

Con el fin de la Guerra, desaparecieron las primas extraordinarias que se habían instituido en numerosos países. Hubo, por tanto, un reajuste en el sector asegurador, tanto en España como en Argentina, que en el caso de Providencia se tradujo en una merma de más de la mitad de los ingresos brutos por contratos de transporte marítimo. Cayó también el interés de compañías más potentes por reasegurar esta parte del negocio de la aseguradora porteña: del 94\% al 60\%. De este modo, hacia 1947, Providencia había redirigido su interés al ramo de incendios, que le aportaba el doble de ingresos respecto del transporte por mar. Entre ambos rubros se colocó la especialidad en accidentes del trabajo, segunda en volumen de negocio.

En este contexto de readaptación, que en el caso argentino incluyó además la puesta en marcha en 1948 del Instituto Mixto de Reaseguros para articular un mercado nacional específico del sector, ${ }^{18}$ Providencia pasó a manos de Generali ese mismo año, cuando la multinacional quiso superar los "efectos catastróficos" que la implicación de Italia en la guerra había tenido para sus actividades y volvió entonces su atención hacia América Latina. En 1939, Generali había llegado al cénit de su expansión internacional, con presencia en 40 países y al frente de un grupo de más de medio centenar de compañías afines. Pero la aventura bélica del régimen fascista italiano se tradujo en la

\footnotetext{
${ }^{15}$ La Gaceta Económica, marzo de 1947, pp. 12345-12346. BCRA-BT.

${ }^{16}$ La Información, mayo de 1942, p. 57, y junio de 1943, pp. 35-36. BCRA-BT.

17 La Gaceta Económica, marzo de 1947, pp. 12345-12346.

${ }^{18}$ Para este organismo, que en 1952 derivaría en el Instituto Nacional de Reaseguros al nacionalizarse de pleno el sector, puede consultarse Zappino (2007).
} 
desconexión con las oficinas ubicadas en países enemigos y en un deterioro creciente de las relaciones con las sucursales de Estados neutrales (caso de España). Cuando la proclividad a nacionalizar el sector asegurador comenzó a extenderse en los primeros años de posguerra por Europa central y oriental, donde Generali había desplegado una parte sustantiva de su red, la compañía determinó expandirse hacia América. Generali adquirió así la mayor parte de Providencia y abrió una sucursal propia en Buenos Aires. El vínculo llega hasta la actualidad, puesto que la empresa opera desde 1994 bajo la denominación de Generali Argentina. Adicionalmente, Generali superó el cambio de siglo como uno de los diez mayores grupos multinacionales aseguradores en América Latina. $^{19}$

Al observar el funcionamiento paralelo de la matriz española que en 1943 se hizo con Providencia, es claro que CHASYR vivía entonces su mejor momento. Era la tercera empresa del país tanto en beneficio financiero como en recaudación de primas, volcada al transporte por mar. Esta especialización -muy rentable por la baja siniestralidad- fue clave para que CHASYR cuatriplicara sus ingresos en 1942 y planteara profundizar la internacionalización del negocio, con una expansión preliminar a Portugal, Marruecos y Chile. Aún pervivía esa idea recién terminada la guerra, cuando se anunciaron "nuevas e interesantes realizaciones en el terreno internacional" para "afianzar la vasta organización creada". ${ }^{20}$ Un entramado que, de asumir la interpretación de Félix Manent (2003, pp. 153-154), se había levantado en apenas un lustro con un propósito doble: obtener la rentabilidad esperada de todo negocio y cobijar bajo el pabellón español las actividades de Generali en tanto continuara la guerra y los intereses italianos en España, sin ser formalmente intervenidos, permaneciesen enrarecidos.

CHASYR se había constituido en 1940 a partir de una pequeña aseguradora local de Castellón (en la costa mediterránea de España, colindante con Cataluña). Aunque su sede se fijó en Madrid, la propiedad de la empresa quedó en manos de un grupo catalán, bajo el liderazgo de la familia Millet y con participación destacada de Josefina Coll, esposa del entonces ministro Carceller. La mitad del capital suscrito se la reservaron los Millet, por un tercio de Coll. Aunque la correlación de fuerzas cambió algo tras una ampliación planteada en 1942, no varió el carácter eminentemente catalán de la compañía (alrededor del $90 \%) .{ }^{21}$ El peso de la gestión lo llevó de hecho Félix Millet i Maristany, un hombre de la banca que trabó relación con Generali a partir de una aseguradora familiar y que en 1936 (iniciada la Guerra Civil) se convirtió en delegado de la multinacional para España. Esta había accedido al mercado español en 1920, al adquirir más de la mitad del capital de otra entidad muy activa en el sector: el Banco Vitalicio de España. Tras la Guerra, Generali transfirió a Millet al menos un 25\% de sus acciones de esta compañía. Esto determinó que CHASYR se desprendiera de varios ramos de actividad, que fueron fusionados con el Vitalicio, para mantener su especialización en transportes. Al mismo tiempo, el grupo internacional nucleado desde CHASYR siguió expandiéndose por Europa. ${ }^{22}$

\footnotetext{
${ }^{19}$ Tortella (2014); Rosasco (2015); http://providencia.com.ar/about.html.

${ }^{20}$ Faes (2020, pp. 216-219); La Vanguardia Española, 6 de julio de 1946, p. 2. Para profundizar en el contexto, véase Tortella (2014).

${ }^{21}$ Lista de accionistas de CHASYR. s.f. Doc. 6254. Fundación Nacional Francisco Franco (FNFF), Madrid, España.

${ }^{22}$ Manent (2003). Para ampliar información sobre Vitalicio, véase Tortella (2014).
} 
El proceso se complicó hacia 1950, en el contexto de la recuperación general de posguerra. Sabemos que Millet envió a Sudamérica en 1951 a uno de sus ejecutivos, Josep Ferrer Bonsoms, "porque algunos países habían bloqueado el dinero que debían a sus compañías" (Canals, 2006, p. 611), y que al menos hasta 1956 este enviado prolongó sus gestiones en Argentina. Como corolario del proceso a largo plazo, CHASYR permaneció bajo control mayoritario de la familia Millet hasta que, en 1988, la aseguradora británica Eagle Star tomó el 100\% de su capital (Tizón, 1988). Transacciones posteriores motivaron que el negocio pasara al grupo suizo Zurich al filo ya del siglo XXI. En cuanto a Vitalicio, esta marca quedó absorbida por completo por Generali Seguros en 2010.

Podremos describir la red que pretendemos caracterizar a partir del cambio en el directorio de la aseguradora porteña en 1943. Al superponer este con el de la textil SADEMA en 1947 (a la que nos hemos referido en el apartado 2), resulta un entramado inmediato con 16 actores individuales más 2 grupales, 150 vínculos reales entre ellos y una centralidad de grado medio -el promedio de vínculos directos trazados por cada actor con el resto- de $8,3 .^{23}$ Veamos quiénes integraban esa red y qué implicaciones tenían en ella.

\section{Etnicidad, coherencia ideológica y conexión a la red corporativa}

En un primer momento, la representación de la familia Millet en Providencia la ostentó Juan Millet Maristany, que actuaba como vocal y representaba, en la periferia del dibujo general, la conexión transnacional más acusada. El papel de delegado de la casa matriz lo desempeñó, sin embargo, Javier Clavell Borrás (Mataró, Barcelona, 1914), un joven abogado especialista en seguros que se incorporó a CHASYR como accionista menor y secretario-director. Clavell, miembro de una familia ligada a Félix Millet en el ámbito de la militancia social-católica, se trasladó a Buenos Aires en febrero de 1945. Despuntó por su competencia técnica, que le valió la representación de Argentina en la Primera Conferencia Hemisférica de Seguros promovida por Estados Unidos en Nueva York, en 1946, para evaluar el sector desde una óptica panamericana. ${ }^{24}$

Abogados eran también, aunque con un prestigio mucho mayor, otros dos de los nuevos directivos de Providencia: Pablo Calatayud (nacido en Montevideo en 1889, de padres argentinos) y Alejandro Gallart Folch (barcelonés cuatro años menor, asentado en Buenos Aires recién terminada la Guerra Civil española). ${ }^{25}$ Al primero se le encomendó la presidencia y el segundo fue designado secretario. Los dos coincidían en su condición de profesores universitarios asomados al mundo de los negocios. Ambos habían militado activamente en política, desde ideologías conservadoras de matriz católica. Gallart fue diputado a Cortes por Barcelona entre 1933 y 1936 (el período en el que la Segunda República española viró a la derecha) como representante de la Lliga Regionalista catalana, una opción autonomista conservadora cuyos cuadros se

\footnotetext{
${ }^{23}$ La fuente básica para el ejercicio es la información contenida en La Información y Guía de Sociedades Anónimas, de Responsabilidad Limitada y Cooperativas, tomando como referencia el bienio 1946-1947. Se incluyen en el análisis aquellos directivos de origen catalán que coinciden en al menos dos empresas, más los partners locales implicados en la parte específicamente financiera del negocio a través de Providencia.

${ }^{24}$ Quién es quién en la Argentina. Biografías contemporáneas (1947, p. 161). BCRA-BT; Centro de Estudios Migratorios Latinoamericanos (CEMLA), s.f.

${ }^{25}$ CEMLA, s.f.
} 
alinearon con el bando sublevado en la Guerra Civil. ${ }^{26}$ Hasta su marcha a Argentina, desempeñó cargos administrativos relacionados con el mundo del trabajo, donde se ubicaba su especialización jurídica, incluida una asesoría al gabinete de Franco en los primeros compases de la guerra. ${ }^{27}$ Ya en Buenos Aires, asumió la representación de la empresa barcelonesa Hispano-Suiza Fábrica de Automóviles, cuyo consejero delegado era uno de sus hermanos, José Gallart Folch. Como tal, Alejandro Gallart recibió en 1944 el encargo de Perón, entonces ministro de la Guerra, para tantear a Franco sobre "una entidad industrial mixta, del Estado Argentino y de la Empresa Española, que con material español y bajo dirección técnica española crearía una fábrica de motores de aviación en este país." ${ }^{28}$ La iniciativa no llegó a materializarse, pero Gallart conservó a priori canales de acceso al Poder Ejecutivo, si sumamos la relación personal que mantenía con José Figuerola desde finales de los años 1920, cuando los dos coincidieron como docentes en la Escuela Social de Barcelona. ${ }^{29}$ Además de sumarse a Providencia, hacia 1947, Gallart era al menos presidente de la importadora Comercial Atlántica, directivo de Samider Financiera, Mercantil, Inmobiliaria, Editorial y de representaciones, y vicepresidente de la Compañía Uruguaya de Representaciones, Importaciones y Exportaciones (CURIE), radicada en Montevideo. Se situaba en un nivel medio-alto de centralidad en la red.

La implicación política y societaria de Pablo Calatayud resulta aún más visible. Seguidor de la tradición jurídica católica argentina, fue seleccionado por el general José Félix Uriburu para intervenir el gobierno de la provincia de Catamarca (al noroeste del país) tras el golpe de Estado que este lideró en 1930, y en 1931 fue elevado a ministro de Obras Públicas. Se adhirió al imaginario protofascista del uriburismo, que apelaba al "pueblo sano" y a "los buenos argentinos" para "eliminar con firmeza lo indeseable" e instalar en el Gobierno a "los más idóneos, los más morales, los más prestigiosos y los más patriotas." ${ }^{\prime 30}$ El declive temprano del proyecto guiado por Uriburu, y la dilución de una solución nacionalista genuinamente argentina en la década de 1930 (Zanatta, 1996; Finchelstein, 2002) dictaron el repliegue de Calatayud a sus negocios particulares. Su cargo de primer presidente de Providencia tras la remodelación de la aseguradora lo concilió, ya mediados los años 1940, con la presidencia de otras siete sociedades, la dirección de dos más y la supervisión interna, como síndico, ${ }^{31}$ de una docena de empresas adicionales. Veintidós compañías en total, de múltiples ramos. ${ }^{32}$ Calatayud fue, por tanto, el gran conector de la red catalana con la red corporativa argentina. O, lo que es lo mismo, fue el actor con una mayor centralidad de grado global (por el que se derivaba un mayor número de conexiones locales hacia la red principal).

\footnotetext{
26 Serie Documentación Electoral, 139, nº 8. Archivo del Congreso de los Diputados, Madrid, España. https://www.congreso.es/web/guest/historico-diputados

27 Quién es quién en la Argentina. Biografías contemporáneas, 1947, p. 258.

${ }^{28}$ Carta de Alejandro Gallart a Francisco Franco. Buenos Aires, 16 de julio de 1944. Doc. 9400. FNFF.

29 La Vanguardia Española, 22 de octubre de 1929, p. 7.

${ }^{30}$ Caras y Caretas, 4 de octubre de 1930, p. 10. HDBNE.

${ }^{31}$ La figura del síndico en las sociedades anónimas argentinas, concebida para que uno de los accionistas designado por la asamblea- fiscalizara la administración, resultó fallida. Para ampliar este asunto, véase Lluch, Rinaldi, Salvaj y Vasta (2019).

32 Guía de Sociedades Anónimas, Responsabilidad Limitada y Cooperativas, 1945-1946. Biblioteca Nacional Mariano Moreno (BNMM). Buenos Aires, Argentina. Providencia contrató el principal espacio publicitario en esta edición, en el canto formado por las páginas de la guía.
} 
Sin embargo, a un nivel interno, la persona que dispuso de más información y que pudo resultar más influyente en el entramado étnico que analizamos fue Antonio Maura Gamazo, con un indicador de centralidad dos veces superior a la media (17 vínculos directos con otros actores). Contaba entonces con 58 años de edad. Hijo del presidente del gobierno español Antonio Maura Montaner -y de raíces por ende mediterráneas, ya que la familia procedía de la isla de Mallorca-, había nacido en Madrid y desde 1917 era cónyuge de Sarah Escalante, hija del exministro argentino Wenceslao Escalante. De ideología monárquica, elitista y conservadora, pero de mentalidad liberal en el mundo de los negocios, se había trasladado a Argentina en 1913. Se colocó en la casa Franklin \& Herrera, comisionada en Buenos Aires por la empresa colonizadora de Guatraché (ubicado al sureste de la región pampeana). ${ }^{33}$ La gestión de las explotaciones agrícolas de su familia política, primero, y la obtención de la representación de la naviera Trasatlántica Española en Argentina, más tarde, le permitieron impulsar un holding propio a finales de los años 1920 al que denominó Maura y Cía. Fue el creador, sobre terrenos familiares en Pilar (en el actual límite norte de la ciudad de Buenos Aires), del Club Tortugas, concebido como un centro de ocio selecto para la práctica de la natación o del polo, y constituido como sociedad anónima en 1930, con participación del entonces presidente Uriburu. La habilitación de chalets para el disfrute de los socios, sobre el diseño de una urbanización cerrada, ha llevado a que se lo considerara el primer country del país (García Rozada, 1996, pp. 9-23). En el momento de su nombramiento como vicepresidente segundo de la aseguradora Providencia, Maura era además primer vicepresidente de la Cámara Española de Comercio en la República Argentina, ${ }^{34}$ se había destacado como promotor, entre otras iniciativas, de una empresa textil creada en 1931 y que definiría así al cierre de la primera presidencia de Perón:

Yo soy aquí presidente y fundador con Santiago Güell de una Cía. que se llama Formio Argentino, que cultiva una planta textil, el Formio [sic] tenax, en una cantidad de islitas que tenemos diseminadas en el Delta del río Paraná. Y en la costa, ya sobre lo que podríamos llamar el continente, está la fábrica donde se decortican las hojas, se batanan y se hila la fibra sola o mezclada con otras fibras que importamos. A esto hemos añadido ahora una instalación completa para hilado de lana... Este negocio, en el que también está [la sociedad] Arnús-Garí, estaba Cambó y algunos amigos más, después de unos años de lucha está siendo muy próspero. ${ }^{35}$

Para manejar sus negocios principales y mantenerlos a una escala de control asequible, Maura se alió con otro inmigrante español con quien venía compartiendo la representación de la Trasatlántica, José Coll Mirambell, nacido en Barcelona hacia $1890 .^{36}$ Juntos promovieron, ya durante la Segunda Guerra Mundial, un holding común

\footnotetext{
${ }^{33}$ Cartas de Antonio Maura Gamazo a Antonio Maura Montaner y Constancia Gamazo. Buenos Aires, 3 de mayo y 3 de junio de 1913. Correspondencia familiar, Caja 113, Legajo 5. Fundación Antonio Maura (FAM). Madrid, España.

34 Información Hispano Argentina, abril-mayo-junio de 1947, s.p. BCRA-BT.

35 Carta de Antonio Maura Gamazo a Gabriel Maura Gamazo. Buenos Aires, 20 de febrero de 1951. Correspondencia 1948-1951, Caja 6, Legajo 4. FAM.

${ }^{36}$ A riesgo de equivocarnos, a la vista de la documentación disponible, interpretamos que José Coll Mirambell es el "José Coll" que desde al menos 1926 compartía con Maura la representación de la Trasatlántica Española en Buenos Aires. Caras y Caretas, 8 de enero de 1927, p. 8. Esto sugiere que podría
} 
que era continuación de la casa Maura y Cía. y que se reconvirtió en la razón Maura y Coll, para atender tres ámbitos paralelos: inmobiliario, cerealista y de navegación. Socio nuclear, pues, de Antonio Maura, José Coll se sumó junto con este al directorio de Providencia, en condición de vocal. De Coll sabemos que ya desde los primeros años de 1920 mantenía relaciones con el entorno de la aseguradora Vitalicio ${ }^{37}$ y que, tras su traslado a Argentina, secundó a Maura prácticamente en todos sus negocios, además de ocupar una primera línea en la movilización profranquista al frente de la Casa de España en Buenos Aires, creada en 1940 (Ferreyra, 2020). Las actividades de esta entidad, de corto recorrido, las respaldaron también Gallart y Maura, quienes ocuparon respectivamente un cargo administrativo y una posición de apoyo secundario a través de la casa Maura y Coll. Sin alcanzar el nivel de centralidad de Maura en la red étnica de intereses catalanes, Coll se le aproximó bastante (14 vínculos directos).

Cuatro nombres más completan el primer organigrama directivo de Providencia tras su reconfiguración. Dos están muy relacionados con la Bolsa de Comercio de Buenos Aires. La primera vicepresidencia se le encomendó a Alejandro Ávalos, vicepresidente de la Bolsa y miembro de la Comisión Nacional de la Marina Mercante. Para el papel de síndico se designó a una de las figuras más reputadas en las finanzas locales: José P. Hernández Lonné, nacido en la ciudad de Buenos Aires en 1891, presidente de la sociedad de valores porteña desde 1946 y comisionista bursátil especializado en cambios, con una larga trayectoria en los mercados financieros. ${ }^{38}$ Ambos proporcionaron un anclaje de la red a los circuitos financieros locales, en los dos casos con niveles de centralidad local reducidos.

La lista original de Providencia se cierra con dos empresarios ligados al sector textil. El joven Pablo Riera Sala, nacido en Barcelona hacia 1908, directivo de las sociedades Textil Alfa y Samider, y próximo al entorno de Félix Millet a propósito de distintas iniciativas del catalanismo cultural (Manent, 2003), fue designado tesorero. Atrajo sobre sí una proporción alta de vínculos en la red, al mismo nivel de centralidad local de Coll y algo por detrás de Maura. Para la vocalía restante de la aseguradora se eligió al industrial de origen italiano Gustavo Malan, de 55 años, ejecutivo de la firma Midlantex, miembro activo de la Cámara de Comercio Italiana en la República Argentina y eventual conector con el empresariado ítalo-argentino. ${ }^{39}$

Seis de esos nueve directivos originales -excluimos a Javier Clavell, que no se incorporaría formalmente hasta 1946- seguían al frente de Providencia en 1947, en vísperas de su adquisición por Generali: Calatayud, Maura, Gallart, Hernández, Riera y Malan. ${ }^{40}$

En el esquema general de la red, es la textil Formio Argentino (además de la capacidad conectora de Calatayud) la sociedad que liga al entramado étnico catalán con los principales grupos empresariales argentinos, en concreto, los conglomerados Bracht y Tornquist. En Formio era también directivo el joven ingeniero barcelonés Evaristo Arnús Moraleda, descendiente de la principal saga de banqueros bolsistas de

tratarse del comerciante catalán homónimo arribado a Buenos Aires a finales de 1917, procedente de Barcelona. CEMLA, s.f.

37 Gaceta de Madrid, 1931, tercer trimestre, p. 134. HDBNE.

38 Quién es quién en Argentina, 1947, pp. 457-458.

39 Al respecto, véase también Guía de Sociedades Anónimas, Responsabilidad Limitada y Cooperativas, 1946-1947, p. 404; CEMLA, s.f.

${ }^{40}$ Guía de Sociedades Anónimas, Responsabilidad Limitada y Cooperativas, 1946-1947, p. 366. 
Cataluña, ingresado a Argentina en abril de 1939. En la medida en que por él pasaron 15 vínculos directos sobre un máximo real de 17, a Arnús le corresponde un nivel alto de centralidad local en la red. En 1943 trabó una conexión adicional con los Tornquist (y con otro gigante corporativo local, el grupo Bunge \& Born) en Raymat SA, una empresa concebida con toda probabilidad para importar el cava catalán producido en origen por la familia Raventós bajo la marca Codorniu. Conector con el grupo Cambó, Arnús mantuvo esas mismas vinculaciones en una pequeña sociedad de inversiones (Inares) creada a mediados de 1947, y las reprodujo en SADEMA. A esta arrastró a uno de sus socios habituales, el también ingeniero Ramón Escayola Canals (de nacionalidad argentina). Escayola y Jesús Raventós, ambos en un nivel de centralidad local algo por encima de la media de la red, contribuyeron a vincularla con el grupo Cambó, como también lo hizo Andrés Bausili, actor clave en el universo corporativo catalán-argentino desde la década de 1920. A su vez, al equipo directivo de SADEMA se aproximó otro de los directivos de Providencia, Pablo Riera, que se integró con Escayola y Arnús en la sociedad Inmobiliaria, Comercial y Financiera, una empresa con participación sustantiva de la familia Cambó. Como segundo ejecutivo de Textil Alfa, Riera compartió además intereses con el industrial Crisanto Ollé, presente también en el directorio de SADEMA. Nacido en Barcelona hacia 1905, Ollé se embarcó para Argentina a finales de 1938, fecha que coincidió con el inicio de la definitiva ofensiva franquista sobre Cataluña. ${ }^{41}$

En la periferia de la red, con bajos niveles de centralidad local, se sitúan por último el exministro y cabeza visible de la comitiva catalana que visitó Buenos Aires en 1947, Demetrio Carceller -alejado de Falange, partidario del autoritarismo personalista encarnado por Franco- (Faes, 2020) y José Bau Nolla (de raíces familiares tradicionalistas, hermano de uno de los técnicos que Franco había reclutado para su primer embrión de gobierno, Joaquín Bau). José Bau era el "primogénito del conocido y acaudalado exportador de aceites y cónsul de las Repúblicas Argentina y Uruguaya" en la localidad catalana de Tortosa, y líder él mismo de un grupo de presión articulado para dar fluidez a las exportaciones hacia Argentina. Consta que ingresó al país por primera vez en $1946 .^{42}$

Cuadro 2. Directivos coincidentes en empresas de la red y/o grandes conectores, con expresión de sectores de actividad en común.

\begin{tabular}{|l|c|c|}
\hline Directivo & Empresas en común & Sectores en común \\
\hline Antonio Maura Gamazo & $\begin{array}{c}\text { Providencia, Formio Argentino, } \\
\text { Naviera Maura y Coll, Inmobiliaria } \\
\text { Maura y Coll, Cerealista Maura y Coll }\end{array}$ & $\begin{array}{c}\text { Seguros, textil, navegación, } \\
\text { inmobiliario, agroexportador }\end{array}$ \\
\hline José Coll & $\begin{array}{c}\text { Providencia, Formio Argentino, } \\
\text { Naviera Maura y Coll, Inmobiliaria } \\
\text { Maura y Coll, Cerealista Maura y Coll }\end{array}$ & $\begin{array}{c}\text { Seguros, textil, navegación, } \\
\text { inmobiliario, agroexportación }\end{array}$ \\
\hline Pablo Calatayud & $\begin{array}{c}\text { Providencia, conector con red } \\
\text { corporativa argentina }\end{array}$ & Seguros \\
\hline Demetrio Carceller Segura & SADEMA, conector con red & Textil \\
\hline
\end{tabular}

41 La Información, octubre de 1947, p. 24, y enero de 1948, p. 15. Guía de Sociedades Anónimas, Responsabilidad Limitada y Cooperativas, 1946-1947, pp. 242, 271-272, 369; Diccionario biográfico de hombres de negocios. Biografías contemporáneas, 1945, pp. 46-47. BNMM; Giralt (2006); CEMLA, s.f. Para los grupos Bunge \& Born y Tornquist, y su desenvolvimiento entre pares, véanse Gilbert (2003), Ceva (2009), Barbero y Lluch (2015).

42 La Vanguardia, 8 de enero de 1918, p. 4, y 2 de febrero de 1934, p. 16. 


\begin{tabular}{|c|c|c|}
\hline & corporativa española & \\
\hline Juan Millet Maristany & $\begin{array}{l}\text { Providencia, conector con Grupo } \\
\text { Millet }\end{array}$ & Seguros \\
\hline Alejandro Gallart Folch & Providencia, Samider & $\begin{array}{c}\text { Seguros, editorial, aeronáutico, } \\
\text { comercio de exportación }\end{array}$ \\
\hline Pablo Riera Sala & Providencia, Samider, Textil Alfa & Seguros, textil, editorial \\
\hline Crisanto Ollé Pérez & Textil Alfa, SADEMA & Textil \\
\hline Evaristo Arnús Moraleda & $\begin{array}{c}\text { SADEMA, Formio Argentino, Raymat, } \\
\text { Inares, Inmobiliaria Comercial y } \\
\text { Financiera }\end{array}$ & $\begin{array}{l}\text { Textil, finanzas, inmobiliario, } \\
\text { comercio, alimentación }\end{array}$ \\
\hline Andrés Bausili Sanromá & $\begin{array}{c}\text { SADEMA, conector con el Grupo } \\
\text { Cambó }\end{array}$ & Textil, finanzas \\
\hline Ramón Escayola Canals & $\begin{array}{c}\text { SADEMA, Inares, Raymat, Inmobiliaria } \\
\text { Comercial y Financiera }\end{array}$ & Textil, finanzas, alimentación \\
\hline Jesús Raventós Fatjó & SADEMA, Inares, Raymat & Textil, finanzas, alimentación \\
\hline Javier Clavell Borrás & Providencia & Seguros \\
\hline José Bau Nolla & SADEMA & Textil \\
\hline Gustavo Malan & $\begin{array}{l}\text { Providencia, conector con red ítalo- } \\
\text { argentina }\end{array}$ & Seguros \\
\hline José P. Hernández Lonné & $\begin{array}{c}\text { Providencia, conector con red } \\
\text { corporativa argentina }\end{array}$ & Seguros \\
\hline
\end{tabular}

Fuente: Elaboración propia a partir de Guía de Sociedades Anónimas, Responsabilidad Limitada y Cooperativas, y La Información. Años 1946-1947.

Proponemos a continuación algunos indicadores para una valoración somera de las principales sociedades en el entorno de la red, en el bienio 1946-1947..$^{43}$ La más antigua era Formio Argentino (1931), la siguió Textil Alfa (escriturada en 1937, aunque reordenada en 1941). Durante la guerra se pusieron en marcha, o se revitalizaron a partir de estructuras previas, la mayoría de las empresas que abordamos: Inmobiliaria, Comercial y Financiera (1939); las tres compañías nucleares de Maura y Coll (19401942); Providencia, Midlantex, Raymat, Comercial Atlántica y Exportadora de Productos Tánicos (1943); y Samider, vinculada al sector editorial (autorizada en 1944). Tras el retorno a la paz, SADEMA y la financiera Inares comenzaron a funcionar a mediados de 1947.

Por capital autorizado, SADEMA fue la primera de la lista 6 millones de pesos en 1947), al mismo nivel de otra firma del ramo, Textil Alfa, y algo por delante de una tercera, Formio Argentino (5 millones). Providencia se situó en un nivel medio respecto del conjunto de las empresas de la red, con 2 millones de pesos. Similares fueron las expectativas planteadas para las tres firmas de Maura y Coll (1-1,5 millones) y la alimenticia Raymat (1,2 millones). Por debajo del millón se ubicaron Comercial Atlántica e Inares. A excepción de las empresas textiles (y excluyendo entre ellas a SADEMA, recién escriturada), fue común que en el momento que tomamos como referencia existiera un desajuste entre el techo de capital establecido (autorizado) y el nivel de capitales realmente interesados en el negocio (suscrito), en proporciones similares o inferiores al $50 \%$ del segundo en relación con el primero.

Finalmente, se constata que las empresas textiles encabezaron a gran distancia el ranking de acumulación de beneficios. Textil Alfa atesoraba unas ganancias de 1,7

\footnotetext{
43 Los datos interpretados en los párrafos siguientes están tomados de la Guía de Sociedades Anónimas, Responsabilidad Limitada y Cooperativas, 1946-1947. Consignamos información del último balance anual disponible de cada empresa.
} 
millones de pesos en 1946-47, seguida de Midlantex (1,5 millones) y Formio Argentino (casi 1,2 millones). Muy por detrás de estas cifras se situaban el resto de firmas para las que disponemos de datos, con sumas, en todo caso, por debajo del umbral de los 100.000 pesos.

\section{Consideraciones finales}

Con una mecánica similar a la que desde finales del siglo XIX venían siguiendo empresas y grupos empresariales ligados a otros países europeos, la iniciativa privada española alcanzó un grado notable de penetración en la economía argentina en los albores del peronismo. En ese flujo transnacional de personas y capitales destacaron equipos directivos específicamente catalanes, al menos en una parte sustancial, que ofrecieron respuestas racionales al contexto resultante de la Segunda Guerra Mundial. Para ello mantuvieron un alto grado de etnicidad que facilitó el control y la ramificación de los negocios, aliados con socios locales entre los que figuraron inmigrantes catalanes previamente establecidos y otros grandes conectores con la red corporativa argentina. Más allá de primar perfiles meramente especulativos, esos directivos promovieron joint ventures con competencia técnica donde fue común la presencia de abogados especializados e ingenieros copartícipes, y plantearon iniciativas conjuntas con vocación de pervivencia. Industria y finanzas fueron ámbitos preferentes de actuación. Siguieron así la estela de un proceso previo de apertura del sector financiero argentino a capitales extranjeros, y participaron de la promoción industrial característica del primer peronismo. Más dinámicos en la creación de empresas hacia la mitad de la guerra, cuando la neutralidad de ambos países abrió espacios a ganancias extraordinarias, los empresarios de origen catalán que ampliaron su radio de acción a Buenos Aires -o fortalecieron allí actividades previas- reforzaron su apuesta por la industria textil en su búsqueda de soluciones adaptativas. No parecieron obedecer a estímulos estatales precisos, si bien se beneficiaron de una rentabilidad que el peronismo inicial habilitó en la práctica por partida doble. Al calor de una relación comercial privilegiada con España, Cataluña siguió proveyendo a Argentina de tejidos de algodón en grandes cantidades, al tiempo que la participación de empresarios catalanes en empresas textiles locales les permitió obtener beneficios adicionales en pleno despliegue de la industria algodonera argentina. Aun constreñidos por un marco regulatorio emergente, esos hombres de negocios mantuvieron un grado estimable de actividad en los primeros años de posguerra, momento para el que varios indicadores sugieren un cierto marasmo en el tejido empresarial transnacional con España. Siguieron inscribiendo empresas, aunque a un ritmo menor, prolongaron las operaciones de sociedades que ya existían y, a partir de la clásica vinculación catalana al textil, se sumaron al ciclo diversificador argentino. En todo ese proceso continuaron una lógica propia, y dispusieron de márgenes de autonomía a la sombra de los sucesivos pactos políticos entre Franco y Perón, una vez agotado el tiempo de la CHADE y de Francesc Cambó como referentes estelares de los negocios en el eje Barcelona-Buenos Aires.

Políticamente, la red que presentamos tuvo su coherencia. La integraron en gran parte empresarios conservadores, en la órbita del regionalismo catalán moderado que se había alineado con el franquismo a raíz de la Guerra Civil. Esa proclividad enlazó con referentes autoritarios como las dictaduras de Primo de Rivera en España y de 
Uriburu en Argentina, y con tradiciones social-católicas o específicamente católiconacionalistas. Aunque la red contó con recursos políticos de primera línea -acceso viable a Franco y a Perón, exministros y exdiputados en sus filas-, cultivó en general operativas de segundo plano, propias de momentos de transición y/o incertidumbre. $Y$ replicó, por último, estrategias empresariales a uno y otro lado del Atlántico, con resultados similares, lo que sugiere que las coordenadas en que se desenvolvía la iniciativa privada en ambos países a mediados de los años 1940 no diferían demasiado, pese a la naturaleza dispar del primer peronismo y de un régimen franquista en vías de institucionalización. El caso a tres de las aseguradoras Providencia, CHASYR y Generali ejemplifica este extremo.

\section{Referencias bibliográficas}

1. Alcalde, R. (2006). Cambó a la CHADE: I'inici dolç i el final amarg. Recerques, 5253,

211-230.

https://www.raco.cat/index.php/Recerques/article/view/137836/322591

2. Ballestero, A. (1993). Juan Antonio Suanzes, 1891-1977. La política industrial de la posguerra. Lid.

3. Banco Central de la República Argentina (1948). Memoria anual. Décimotercer ejercicio, 1947. Talleres Gráficos del Banco Hipotecario Nacional.

4. Banco Central de la República Argentina (1949). Memoria anual. Décimocuarto ejercicio, 1948. Talleres Gráficos del Banco Hipotecario Nacional.

5. Barbero, M. I. (2011). Los grupos económicos en la Argentina en una perspectiva de largo plazo (siglos XIX y XX). En G. Jones y A. Lluch (Eds.) El impacto histórico de la globalización en Argentina y Chile: empresas y empresarios (pp. 1-37). Temas.

6. Barbero, M. I. y Lluch, A. (2015). El capitalismo familiar en Argentina: modelos y dinámicas en el largo plazo. En P. Fernández Pérez y A. Lluch (Eds.) Familias empresarias y grandes empresas familiares en América Latina y España: Una visión de largo plazo (pp. 219-260). Fundación BBVA.

7. Belini, C. (2009). La industria peronista, 1946-1955. Políticas públicas y cambio estructural. Edhasa.

8. Belini, C. (2010). El lento desenvolvimiento de la industria textil lanera argentina y la sustitución de importaciones durante la entreguerras, 1914-1939. Investigaciones y Ensayos, 59, 111-142.

9. Belini, C. (2013). Miguel Miranda. El mago, la intuición y la breve prosperidad de la economía peronista. En R. Rein y C. Panella (Comps.) La segunda línea. Liderazgo peronista, 1945-1955 (pp. 243-265). Universidad Nacional Tres de Febrero. 
10. Belini, C. (2014). Convenciendo al capital. Peronismo, burocracia, empresarios y política industrial, 1943-1955. Imago Mundi.

11. Belini, C. y Korol, J. C. (2020). Historia económica de la Argentina en los siglos $X X$ y XXI. Siglo XXI.

12. Berrotarán, P. (2013). José Figuerola. El estadígrafo de Perón. En R. Rein y C. Panella (Comps.) La segunda línea. Liderazgo peronista, 1945-1955 (pp. 175193). Universidad Nacional Tres de Febrero.

13. Cabrera, M. (2011). Juan March (1880-1962). Marcial Pons.

14. Cabrera, M. y Del Rey, F. (2002). El poder de los empresarios: política e intereses económicos en la España contemporánea (1875-2000). Taurus.

15. Cámara de Diputados de la Nación (1941). Comisión Especial Investigadora de las Concesiones de Servicios Eléctricos de la Capital. Informes y Conclusiones. Imprenta del Congreso Nacional.

16. Cámara Española de Comercio en la República Argentina (1948). Memoria anual correspondiente al ejercicio 1947-1948. Imprenta Santos.

17. Campins, M. y Pfeiffer, A. (2011). La importancia de las redes sociales en los orígenes de la industria farmacéutica argentina. El caso de los catalanes en Argentina. Revista de Historia Industrial, 47 (3), 17-50. https://www.raco.cat/index.php/Historialndustrial/article/view/248224/332337

18. Canals, C. M. (2006). Josep Ferrer Bonsoms (1920-2001). En F. Cabana (Coord.) Cien empresarios catalanes (pp. 610-618). Lid.

19. Carreras, A. (1990). Cataluña, primera región industrial de España. En J. Nadal y A. Carreras (Coords.) Pautas regionales de la industrialización española (siglos $X I X Y X X)($ pp. 259-295). Ariel.

20. Catalan, J. (1995). La economía española y la Segunda Guerra Mundial. Ariel.

21. Centro de Estudios Migratorios Latinoamericanos [base de datos] (s.f.). https//cemla.com/buscador/

22. Ceva, M. (2009). De la exportación cerealera a la diversificación industrial. Las empresas Bunge y Born en Argentina (1884-1940). Estudios Migratorios Latinoamericanos, 65, 81-98.

23. Comín, F. y Martorell, M. (2013). La Hacienda pública en el franquismo. La guerra y la autarquía (1936-1959). Instituto de Estudios Fiscales. 
24. Cortés, R. (2006). La economía política de la Argentina en el siglo XX. Edhasa.

25. Dalla Corte, G. (2005). Casa de América de Barcelona (1911-1947). Comillas, Cambó, Gili, Torres y mil empresarios en una agencia de información e influencia internacional. Lid.

26. Dalla Corte, G. (2006). Empresas e instituciones y red social. La Compañía Hispano Americana de Electricidad (CHADE) entre Barcelona y Buenos Aires. $\begin{array}{llll}\text { Revista de } \quad \text { Indias, 519-544. } & \end{array}$ http://revistadeindias.revistas.csic.es/index.php/revistadeindias/article/view/348/ 410

27. De Riquer, B. (2016). Cambó en Argentina: Negocios y corrupción política. Edhasa.

28. Faes, E. (2020). Demetrio Carceller Segura (1894-1968): un empresario en el gobierno. Galaxia Gutenberg.

29. Fernández, A. (2004). Un mercado étnico en el Plata. Emigración y exportaciones españolas a la Argentina (1880-1935). Consejo Superior de Investigaciones Científicas.

30. Fernández, A. (2019). Los catalanes y Buenos Aires: inmigración, asociaciones y prensa. Almaluz.

31. Ferreyra, A. N. (2020). La Casa de España: un fallido proyecto institucional del falangismo en Buenos Aires (1940-1942). Quinto Sol, 24 (3), 1-22. https://doi.org/10.19137/qs.v24i3.4073

32. Figallo, B. (1992). El protocolo Perón-Franco. Relaciones Hispano-argentinas (1942-1952). Corregidor.

33. Finchelstein, F. (2002). Fascismo, liturgia e imaginario: El mito del general Uriburu y la Argentina nacionalista. Fondo de Cultura Económica.

34. García Rozada, N. (1996). Tortugas. s.e.

35. García Sebastiani, M. (Dir.) (2010). Patriotas entre naciones. Élites emigrantes españolas en Argentina. Editorial Complutense.

36. Gerchunoff, P. y Llach, L. (1998). El ciclo de la ilusión y el desencanto. Un siglo de políticas económicas argentinas. Ariel.

37. Gilbert, J. (2003). Entre la expansión y la crisis de la economía argentina. Ernesto Tornquist y Cia. Ciclos, 25-26, 65-92. 
38. Giralt, E. (2006). Manuel Raventós i Domènech (1862-1930). En F. Cabana (Coord.) Cien empresarios catalanes (pp. 243-251). Lid.

39. Jensen, S. (2010). La comunidad catalana en Argentina al arribo de los exiliados de 1939: tensiones y debates en el mantenimiento de la unidad nacional. Estudios Migratorios Latinoamericanos, 69, 413-436.

40. Lanciotti, N. (2011). Del Estado garante al Estado empresario. La relación entre Estado y empresas de servicios públicos urbanos en Argentina, 1880-1955. En G. Jones y A. Lluch (Eds.) El impacto histórico de la globalización en Argentina y Chile: empresas y empresarios (pp. 187-217). Temas.

41. Lanciotti, N. (2021). Historia empresarial de Argentina: empresas familiares, grupos económicos y multinacionales extranjeras en Argentina (1875-2010). En A. Lluch, M. Monsalve y M. Bucheli (Eds.) Historia empresarial en América Latina: temas, debates y problemas. Universidad del Pacífico/Universidad de Los Andes. Ebook.

42. Lanciotti, N. y Lluch, A. (Eds.) (2018). Las empresas extranjeras en Argentina desde el siglo XIX al siglo XXI. Imago Mundi.

43. Lluch, A., Rinaldi, A., Salvaj, A. y Vasta, M. (2019). Directors and syndics in corporate networks: Argentina and Italy compared (1913-1990). Business History, 61 (4), 603-628. https://doi.org/10.1080/00076791.2017.1382474

44. Lluch, A. y Salvaj, E. (2018). La red corporativa argentina y el rol de las empresas extranjeras: un estudio desde las redes de directorios (1923-2000). En N. Lanciotti y A. Lluch (Eds.) Las empresas extranjeras en Argentina desde el siglo XIX al siglo XXI (pp. 217-233). Imago Mundi.

45. Lucci, M. (2020). El "seny" y la "rauxa" en la revista Ressorgiment durante la guerra civil española. Los "catalanes de América" de Buenos Aires y la defensa de la II República española desde el exilio (1936-1939). En N. De Cristóforis (Dir.) Los españoles en Buenos Aires. Activismo político e inserción sociocultural (1870-1960). https://teseopress.com/espanolesenbuenosaires

46. Maluquer, J. (2006). Prólogo. En F. Cabana (Coord.). Cien empresarios catalanes (pp. 9-10). Lid.

47. Manent, A. (Dir.) (1992). Diccionari dels catalans d'Amèrica. Contribució a un inventari biográfic, toponímic i temàtic. Generalitat de Catalunya.

48. Manent, A. (2003). Fèlix Millet i Maristany: Líder cristiá, financer, mecenes catalanista. Proa. 
49. Martínez-Garralaga, J. y Prat, M. (2016). Wages, prices and technology in early Catalan industrialization. The Economic History Review, 69 (2), 548-574. https://doi.org/10.1111/ehr.12127

50. Monner, R. (1927). Los catalanes en la Argentina. Coni.

51. Moya, J. C. (2004). Primos y extranjeros. La inmigración española en Buenos Aires, 1850-1930. Emecé.

52. Núñez Seixas, X. M. (2014). Las patrias ausentes. Estudios sobre historia y memoria de las migraciones ibéricas (1830-1960). Genueve.

53. Puig, N. (2006). La empresa en Cataluña: identidad, supervivencia y competitividad en la primera región industrial de España. En J. L. García Ruiz y C. Manera (Dirs.) Historia empresarial de España. Un enfoque en profundidad (pp. 27-56). Lid.

54. Rein. R. (2003). Entre el abismo y la salvación. El pacto Franco-Perón. Lumiere.

55. Rocamora, J. (1992). Catalanes en la Argentina. En el centenario del Casal de Catalunya. El Fénix.

56. Rocamora, J. (1995). Records d'un exiliat a Amèrica. Rafael Dalmau.

57. Rosasco, R. (Ed.) (2015). The Age of the Lion. Assicurazioni Generali's long journey from 1831 to the third Millennium. Assicurazioni Generali.

58. Rougier, M. (2007). Crédito e industria en tiempos de Perón, 1944-1955. Revista $\begin{array}{llll}\text { de Historia } & \text { Industrial, } & \text { 79-113. }\end{array}$ https://www.raco.cat/index.php/Historialndustrial/article/view/82450

59. Rubio, J. A. y Garrués, J. (2016). Economic and Social Power in Spain: corporate networks of banks, utilities and other large companies (1917-2009). Business History, 58 (6), 858-879.

60. Rubio, J. A. y Garrués, J. (2018). La red corporativa española en el largo plazo. Evidencia de una economía de mercado influenciada por el Estado. Revista Internacional de Sociología, 76 (3). https://doi.org/10.3989/ris.2018.76.3.16.174

61. San Román. E. (1999). Ejército e industria: el nacimiento del INI. Crítica.

62. Sarramone, A. (2004). Cataluña y los catalanes en el Plata. Biblos.

63. Tizón, Á. "Eagle Star se hace con el 100\% de Chasyr". El País, 23/2/1988. https://elpais.com/diario/1988/02/24/economia/572655611_850215.html 
64. Tortella, G. (Dir.) (2014). Historia del seguro en España. Fundación Mapfre.

65. Vidal, J. (2011). El crecimiento y expansión de las empresas y la inversión española en Argentina y el cono sur en el siglo XX. En G. Jones y A. Lluch (Eds.) El impacto histórico de la globalización en Argentina y Chile: empresas y empresarios (pp. 133-154). Temas.

66. Yrurzun, J. (2017). Redes de sociabilidad artística y cultural. El activismo de los catalanes de Buenos Aires (Argentina, inicios del siglo XX). En A. Reguera (Dir.) Vínculos que configuran redes. Las dimensiones relacionales de lo social y sus articulaciones a escalas diferenciadas (pp. 219-244). Teseo.

67. Zanatta, L. (1996). Del Estado liberal a la nación católica. Iglesia y Ejército en los orígenes del peronismo. Universidad Nacional de Quilmes.

68. Zappino, J. S. (2007). El Instituto Mixto Argentino de Reaseguros: la formación de un mercado nacional de seguros (1946-1952). Ediciones Cooperativas. 